\title{
Fibrinolysis and carcinoma of the prostate
}

\author{
H. T. SWAN AND D. F. KERRIDGE \\ From the Department of Haematology, Royal Infirmary and Royal Hospital, Sheffield, \\ and Department of Statistics, University of Sheffield
}

SYNOPSIS In a series of 68 patients suffering from carcinoma of the prostate, plasma fibrinolyticis activity has been found to be similar to that in control groups.

Some comments have been made on the problem of fibrinolysis and bleeding associated witlo carcinoma of the prostate, with the suggestion that the part played by fibrinolysis has been over $\overrightarrow{\dot{0}_{0}}$ emphasized.

An association of serious bleeding with prostatic carcinoma has been described from time to time (Jürgens and Trautwein, 1930; Marder, Weiner, Schulman, and Shapiro, 1949; Seale, Jampolis, and Bargen, 1951; Cosgriff and Leifer, 1952) but Tagnon, Whitmore, and Shulman (1952) appear to be the first to have given prominence to fibrinolysis as a cause of such bleeding. They restricted their definition of fibrinolysis to the dissolution within 24 hours of whole blood clot or plasma, kept at $37^{\circ} \mathrm{C}$., and described three examples of spontaneous bleeding associated with fibrinolysis in cases of carcinoma of the prostate. In the subsequent year Tagnon, Whitmore, Schulman, and Kravitz (1953b) collected all their cases, including two new ones, and reported fibrinolysis in six out of 48 patients. This may be the largest such published series.

It is the purpose of this paper to report the results of a study of fibrinolysis made on 68 patients suffering from carcinoma of the prostate.

\section{MATERIALS AND METHODS}

PATIENTS: MAIN SERIES Sixty-eight patients aged 51-87 years were examined. All of them had already been diagnosed as having a prostatic carcinoma. Two of these were investigated for fibrinolysis because of bleeding and bruising. The remaining 66 who showed no obvious bleeding tendency presented over the course of four years and were accepted for study of fibrinolysis as they arose and without selection. In 44 the diagnosis of carcinoma of the prostate was confirmed histologically either at the time or subsequently. In 17 others the diagnosis was beyond reasonable doubt, although without histological proof. In such cases the clinical story and characteristic findings on palpation of the prostate were further supported by the presence of osteoplastic secondary deposits

Received for publication 21 September 1964. in bone or significantly raised serum acid phosphatas levels in the blood. In the seven remaining cases the diag nosis was made clinically and was without strong enough supporting evidence to make the diagnosis unchallengeable. Metastases were known to be present in 22 patientso Although repeated examinations for fibrinolysis werer made in some cases only first examinations are recorded here. All but 12 were in-patients at the time of this test.

PATIENTS: CONTROL SERIES One hundred men in hospita were used as controls. Forty-one were suffering from known malignant disease which was not carcinoma @ the prostate. In the remaining 59 no malignant diseas was suspected. Many of these were in hospital with 9 view to surgery for varicose veins or hernia.

Days of obvious stress, such as their operation da were avoided for all patients whether in the main seriess or in the controls, and with the exception of the 12 whi were out-patients, all were in bed at the time of the venepuncture. This was almost invariably about 10 a.m after the patients had eaten a hospital breakfast.

METHOD The fibrinolytic activity of plasma was mezsured. Assays were carried out by observation of the lysis time of clots prepared from ice-cold fresh plasma an equal volume of sterile veronal buffer at $p \mathrm{H} 7 \cdot 4$. Whe this $50 \%$ plasma was incubated at $37^{\circ} \mathrm{C}$. a clot formeg spontaneously which was then observed at intervals for the time of its dissolution or complete disintegration. $N$

Observation of the clot from $50 \%$ plasma was in faet part of a larger experiment based on the method $\Theta$ Fearnley and Lackner (1955) but it proved difficult to compare results from different experiments using different concentrations of plasma in each. The usu pattern of lysis in such cases was demonstrated when the clot from $10 \%$ plasma lysed first and was followed in sequence by the clots from the nine more concentrated plasmas. However almost one in four experiments showed lysis according to a different pattern (see al\$o Table 1, Fearnley and Tweed, 1953) and this made comparisons difficult. The suitability of the lysis time of 
TABLE

OBSERVED AND EXPECTED INCIDENCE OF LYSIS OCCURRING WITHIN THREE ARBITRARY TIME PERIODS

Numbers of Cases

\begin{tabular}{|c|c|c|c|c|c|}
\hline \multirow{2}{*}{$\begin{array}{l}\text { Lysis } \\
\text { Time } \\
\text { (hours) }\end{array}$} & \multicolumn{2}{|c|}{ Carcinoma of Prostate } & \multicolumn{2}{|l|}{ Controls } & \multirow[t]{2}{*}{ Total } \\
\hline & Observed & Expected & Observed & Expected & \\
\hline $\begin{array}{l}100+ \\
50-99 \\
0-49 \\
\text { Totals }\end{array}$ & $\begin{array}{l}11 \\
25 \\
31\end{array}$ & $\begin{array}{l}11.95 \\
28.23 \\
26 \cdot 82 \\
67\end{array}$ & $\begin{array}{l}19 \\
50 \\
31\end{array}$ & $\begin{array}{r}18 \cdot 05 \\
46 \cdot 77 \\
35 \cdot 18 \\
100\end{array}$ & $\begin{array}{r}30 \\
75 \\
62 \\
167\end{array}$ \\
\hline
\end{tabular}

The $\chi^{2}$ test has been applied allowing for the age differences. $\chi_{2}^{2}=1.8664 \quad P=0.5$ (not significant).

the $50 \%$ plasma clot as representing each experiment was found by a statistical study of all the figures. It was the greatest concentration at which lysis occurred in almost all cases. The same conclusion could be shown graphically by plotting all 10 lysis times in each experiment when it was seen that the lysis time of the $50 \%$ plasma clot was the best single representative of the general rate of lysis in all experiments. When the $10 \%$ or $100 \%$ plasma clots were used, they gave on some occasions an impression of lytic activity which was inconsistent with the rest of the experiment. A more detailed description of the method used has been given elsewhere (Swan, 1963).

Ten lysis times are not available, nine of these being due to failure of the clot to lyse completely even after observation for a week. The effect on the results of the lack of an exact lysis time for nine experiments is discussed later.

\section{RESULTS}

The 61 available lysis times in the main series were arranged in groups by decades according to the ages of the patients. Such an arrangement allows for the shortening of the lysis time associated with age, demonstrable by this method (Swan, 1963). The mean of each group was calculated and has been plotted in Fig. 1 together with twice the standard error on either side. On the same figure the means similarly derived for the control groups have been plotted.

It can be seen from Fig. 1 that there is no significant difference between the lysis times for patients suffering from carcinoma of the prostate and the controls. This remains true whether the two control groups, of malignancy and no known malignancy, are taken separately or together.

A different analysis of the results was then made in order to test further whether there was evidence of increased fibrinolysis in carcinoma of the prostate. Experiments were arranged into three arbitrary groups according to the observed lysis times. The number of cases in each group is recorded in the Table. It can be seen that the number of cases of carcinoma of prostate which lyse in the shortest period shows a slight increase over the 'expected' number but that this is not more than could occur by chance.

It has been suggested by Tagnon and co-workers $(1953 a, b)$ that the source of the fibrinolysin may be tissue juice from the carcinoma itself and that this may be a quantitative matter, being more apparent in cases of metastatic carcinoma where there is the greater volume of pathological tissue. It is also reasonable to assume in most cases that the maximum amount of pathological tissue in any one patient is present in his last few weeks of life. For this reason all the assays from the $\mathbf{3 0}$ patients in the present series who are known to have died within two years of the test have been plotted against the

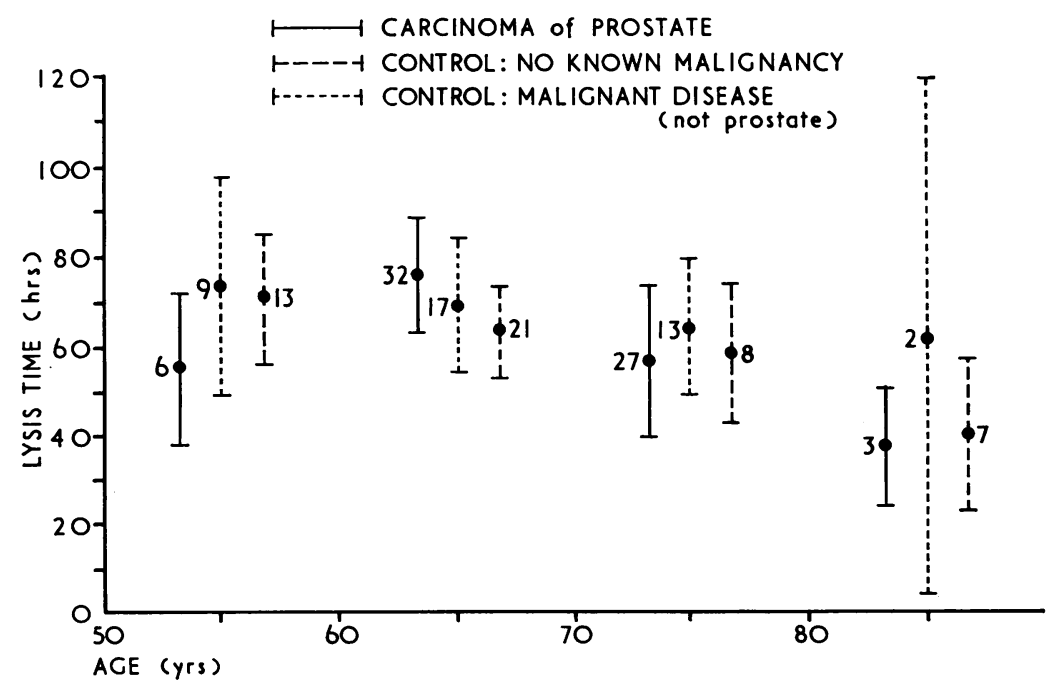

FIG. 1. Mean lysis times of cases of carcinoma of prostate taken by decades of age and shown $\pm 2 \times$ standard errors of the means, together with two control groups similarly treated. The number of cases in each group is indicated. 


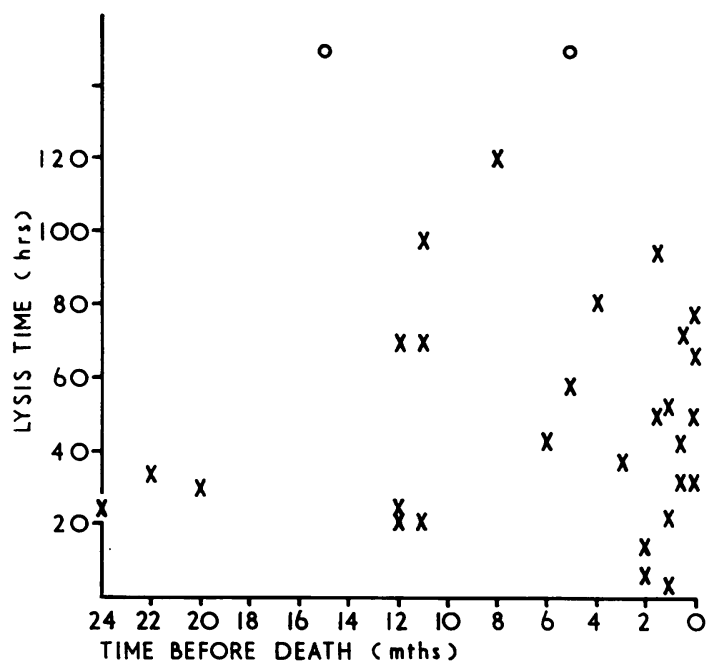

FIG. 2. The lysis times of 30 cases of carcinoma of prostate plotted against the time before death. Small circles represent clots which failed to lyse.

time before death (Fig. 2). It can be seen that there is no obvious clustering of points at any place and in particular the clot lysis time of patients who have no more than a few weeks to live is not obviously shorter than the others.

One of the patients who died justifies further description. His was one of the only two cases where fibrinolysis studies were initiated because of a bleeding state, in this case haematuria and petechiae. His rate of lysis was extremely fast, the whole blood clot having dissolved away within twoand-a-half hours. When the man died four weeks later, necropsy showed a carcinoma of prostate confirmed histologically, but supplied no evidence of metastasis to other parts of the body; nor was there evidence of bleeding. The sternum and the skull were the only bones sectioned. In this case the fast fibrinolysis did not appear to be the product of a large amount of carcinomatous tissue in the body.

\section{DISCUSSION}

When Tagnon and his colleagues (1952) looked for fibrinolysis in 14 consecutive patients known to have carcinoma of the prostate and who were not subject to bleeding at the time, they did not find it. This paper reports a similar vain search in a larger series.

On the results presented here any difference between fibrinolytic activity shown in patients known to have a prostatic carcinoma and the controls is well within the limits of chance variation although we can never exclude the possibility that the use of larger numbers of patients or a more sensitive method of assay might reveal a difference. If there does exist a real difference characteristic of ca cinoma of the prostate, one which would be found in any random group of such cases, then it must be small.

The nine clots which failed to lyse even afte observation for a prolonged time have not been i cluded in the calculations involved in Figure 1. Sư of these were from patients in the main group of prostatic carcinoma and three were from the contri group. It can be seen that if they had been includeed in the calculations by giving them an arbitrar figure representing a prolonged lysis time this coufef only have reinforced the conclusion already reached that carcinoma of the prostate is not associated witi an increased rate of fibrinolysis. The same nine exs periments have been included in the calculations involved in the Table.

Serious bleeding resulting from disordered haemostasis nevertheless remains a real threat in rare casass of carcinoma of the prostate and the problem Is what part, if any, is played in them by fibrinolysifo Some such cases have been found to have a very low level of plasma fibrinogen without demonstrable fibrinolysis (Frick, 1956; Swan, Wood, and Danie 1957; case 2: Rapaport and Chapman, 1959). 届 many other cases concurrent hypofibrinogenaem and fibrinolysis have been demonstrated. On the other hand rapid fibrinolysis without hypofibring genaemia may not be of clinical importance, for this has often been shown to be present without associated bleeding even in the presence of frest surgical wounds (Macfarlane, 1937).

Where there is disordered haemostasis it may that fibrinolytic activity is secondary to intravasci lar coagulation and that fibrinolysis is not itself the cause of the hypofibrinogenaemia by means of digestion of fibrinogen in vivo. Tagnon, Schulman, Whitmore, and Leone (1953a) twice noted evidence of hypercoagulability to precede any increase fibrinolysis in a case of carcinoma of the prostate; and Soulier, Mathey, Le Bolloch, Daumet, anf Fayet (1952) had already demonstrated hypercoagull ability to precede fibrinolysis during the course pulmonary resection.

Tagnon and his co-workers (1953b) found that extract of carcinomatous prostatic tissue shows vefy active fibrinolytic activity in vitro and the observation has been confirmed in this laboratory. This is the basis of Tagnon's hypothesis that such juices a released into the blood stream where they act as powerful lytic agent. However it has been show quite convincingly in this laboratory (Swan, 196\%) that tissue juices extracted from two carcinomato prostates have had thromboplastic activity in ad- 
dition to fibrinolytic activity, and that such juice can act as an efficient substitute for brain residue in the Quick one-stage prothrombin test using normal plasma. It seems therefore that experimental evidence going further than what was supplied is required to substantiate the postulate that carcinomatous tissue juice from the prostate or its metastases is a direct source of plasma fibrinolysin.

Despite the negative findings found here in relation to fibrinolysis, there is no doubt about the severity of the generalized bleeding which can take place in association with carcinoma of the prostate. More detailed investigational work is required on these relatively rare cases.

The authors are indebted to Mr. J. C. Anderson, the late Mr. G. W. Blomfield, Professor R. P. Jepson, Professor A. W. Kay, and other surgeons and physicians under whose care the patients were. Financial help for the purchase of equipment was provided by the Board of Governors of the United Sheffield Hospitals from their endowment fund. Finally thanks are due to Dr. E. K. Blackburn for his cooperation during the work.

\section{REFERENCES}

Cosgriff, S. W., and Leifer, E. (1952). J. Amer. med. Ass., 148, 462. Fearnley, G. R., and Lackner, R. (1955). Brit. J. Haemat., 1, 189. _—, and Tweed, J. M. (1953). Clin. Sci., 12, 81.

Frick, P. G. (1956). Acta haemat. (Basel), 16, 11.

Jürgens, R., and Trautwein, H. (1930). Dtsch. Arch. klin. Med., 169, 28.

Macfarlane, R. G. (1937). Lancet, 1, 10.

Marder, M., Weiner, M., Schulman, P., and Shapiro, S. (1949). N.Y. St.J. Med., $49,1197$.

Rapaport, S. I., and Chapman, C. G. (1959). Amer. J. Med., 27, 144.

Seale, R. A., Jampolis, R. W., and Bargen, J. A. (1951). Surg. Clin. N. Amer. 31, 1111.

Soulier, J. P., Mathey, J., Le Bolloch, A. G., Daumet, P., and Fayet, H. (1952). Rev. Hémat. 7, 30.

Swan, H. T. (1961). Fibrinolysis and its Relationship to Age and to Carcinoma of the Prostate. M.D. Thesis, University of Edinburgh.

(1963). Brit.J. Haemat., 9, 311.

- Wood, K. F., and Daniel, O. (1957). Brit. med. J., 1, 495.

Tagnon, H. J., Schulman, P., Whitmore, W. F., and Leone, L. A. (1953a). Amer.J. Med., 15, 875.

-, Whitmore, W. F. Jr., Schulman, P., and Kravitz, S. C. (1953b). Cancer (Philad.) 6, 63.

$\longrightarrow$, — , and Shulman, N. R. (1952). Ibid., 5, 9. 